\title{
Incidence of Elevated Aminotransferases Levels among Patients Presenting with Hepatitis Syndrome in the Northwest Region of Cameroon
}

\author{
Lem Edith Abongwa*, Nantia Akono, Abea Princewill Elo and Christian Vershiyi Junior \\ Department of Biological Sciences, University of Bamenda, Cameroon
}

*Corresponding author: Lem Edith Abongwa, Faculty of Science, Department of Biological Sciences, University of Bamenda, PO Box 39, Bambili, NW Region, Cameroon

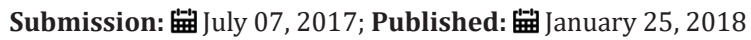

\begin{abstract}
Hepatitis B (HB) and Hepatitis C (HC) are a leading cause of liver enzyme elevations in patients (LEE). This study aimed to estimate the prevalence of LEE among patients with hepatitis syndrome. This study enrolled 192 participants presenting with signs and symptoms of hepatitis in the Northwest Region of Cameroon from August 2015 to May 2016. Venous blood samples were collected for the diagnosis of HBV and HCV. Enzymatic measurement of ALT and AST was done using a biochemical analyzer. ALT and AST were categorised using standardized toxicity grade on upper limit of normal.

The age ranged of the patients was 2-98years with a mean(SD) age of 41.42( \pm 18.04$)$. Of the 192 patients 97(50.5\%) were female, HBV and HCV prevalences were $100(52.1 \%)$ and $0(0.0 \%)$ respectively. The prevalences of HBV were insignificantly $(\mathrm{p}=>0.05)$ higher in female $54(56.8 \%)$ and in the age range $<30 y e a r s 31(58.5 \%)$. ALT and AST activities showed significant $(\mathrm{p}<0.05)$ higher values $76.9 \pm 10.6$ and $106.7 \pm 14.4$, respectively in HBV positive than in HBV negative individuals. The prevalence of elevated ALT and AST enzymes were 68(35.4\%) and 85(44.3\%) respectively. Age and gender-wise prevalances showed high prevalence in $>50$ years and in male. Elevated and significant transaminase activities were found in HBV positive compared to negative subjects. Alcoholic liver disease was common in HBV negative patients while chronic liver disease was higher among HBV positive patients $(\mathrm{P}=0.012)$. Elevated ALT and AST levels are common in both HBV positive and HBV negative subjects indicating multifactorial causes. As such effective management of hepatotoxicity requires accurate diagnosis.
\end{abstract}

Keywords: ALT; AST; HCV; HBV; Hepatotoxicity

\section{Introduction}

Hepatitis B (HB) and Hepatitis C (HC) are the leading causes of chronic liver disease in the world [1]. They are considered as one of World Health Organization (WHO) public health priority after the HIV infection, malaria and tuberculosis [2,3]. Worldwide, it is estimated that more than 2 billion people and 170 million people are infected with hepatitis B virus (HBV) and hepatitis C virus (HCV) respectively with $3-4$ million new infections occurring each year $[4,5]$. The prevalence of $\mathrm{HB}$ and $\mathrm{HC}$ is highest in subSaharan Africa and East Asia, with an estimated $5-10 \%$ adults with chronic infection [2]. Of these, 240 million people are chronic carriers of $\mathrm{HBV}$ and are at risk of death from acute fulminant liver disease, hepatocellular carcinoma (HCC) or liver cirrhosis [5,6]. In Cameroon the prevalence of HBV ranges from 6.3-11.5\% [7] while the range of HCV is $6.9 \%-56.0 \%$ [8-10].

Diagnostic methods for liver diseases include the measurement of serum aminotransferases (alanine aminotransferase [ALT] and asparate amniotransferase [AST]) [11]. Thus their levels increase in chronic hepatitis B and C infections [12,13]. Liver enzyme alteration may either be the accompanying biochemical picture in

a patient with symptoms or signs suggestive of liver disease or an isolated unexpected finding in a patient who has undergone a wide range of laboratory tests for a non-hepatic disease or used for little children [14].

Syndrome associated with $\mathrm{HB}$ and $\mathrm{HC}$ ranges from mild to severe and include abdominal pain, enlarge stomach, dark urine, fever, Muscle or Joint pain, Loss of appetite, Nausea, Vomiting, Fatigue, Weight loss, Clay-colored stool, jaundice and Tenderness around the liver. Injury of the liver by hepatitis virus is caused by the body's immune response as the body attempts to eliminate the virus. As such the level of hepatitis viral load may determine the degree of liver damage $[15,16]$.

The magnitude of liver enzyme elevation (LEE) in the Cameroonian population is currently unknown. This study therefore was conducted to reinforce the significance and etiology of elevated ALT and AST in a population presenting with sign and symptoms of liver disease for proper management of the disease. Furthermore we seek to investigate the type of liver disease that is common in patients presenting with hepatitis syndrome. 


\section{Materials and Methods}

\section{Study design, site and population}

This study was carried out in the Northwest region of Cameroon from August 2015 to May 2016. It was a hospital based crosssectional study which enrolled participants who presented with signs and symptoms of hepatitis in the outpatient department of Mezam Polyclinic and Regional Hospitals. The exclusion criteria were participants who refused to be part of the study, patients who were known to be seropositive for HBV or HCV and those with known HIV positive status. Sample size was calculated based on Raosoft online calculator using the level of significance of 0.05 and power of $80 \%$ assuming that the expected prevalence is $10 \%$. [17] A total of 192 patients of both sexes exceeded the minimum required sample size of 97.

\section{Ethical consideration}

Ethical clearance was obtained from the institution ethical review boards. Clients above 18 years and guardians of children 18 years and below were verbally consented. The aims of the study and procedure to be taken were explained to them and their confidentiality ensured prior to sample collection.

\section{Laboratory procedure}

After venous blood collection, samples were allowed to clot and centrifuged at $3000 \mathrm{rmp}$ for $3 \mathrm{mins}$. HBV and HCV were diagnosed using HBV and HCV Diaspot strips (Jakarta, Indonesia) which detect HBsAg or HCV respectively from the patient serum. ALT and AST were measure enzymatically using biochemical analyzer (Biobase.
CC) according to the Chronolab procedure (Barcelona, Spain). ALT and AST were categorised using standardized toxicity grade scale using the normal range of ALT and AST levels as being on upper limit of normal (ULN) value according to age and sex. Grade 0 (normal; $<1.25$ ULN), Grade 1(mild <1.25-2.5 ULN), Grade 2 (moderate 2.65.0 ULN), Grade 3(severe > 5.0-10 ULN) and Grade 4(life threatening $>10$ ULN) [3]. Non severe hepatotoxicity is considered as grade 1 and 2 toxicity while and severe hepatotoxicity is defined as grade 3 and 4 toxicity level [18].

\section{Data analysis}

Data was enter in excel, double check in Microsoft Excel 2010 and analysed using the Statistical Package for Social Sciences (SPSS), version 16. Continuous variables were summarized as means (Standard Error $\{\mathrm{SE}\}$ ), and categorical variables as frequencies and percentages. The chi-square $(\chi 2)$ test was used to determine the association of elevated ALT and AST levels with age and sex student $t$ test or ANOVA to compare the difference between means. P value of $<0.05$ was considered statistically significant.

\section{Results}

\section{Prevalence study}

Of the 192 patients, 97(50.5\%) were females with a median age of 40.57 years (range 6-76 years) and 95 (49.5\%) males with a median age of 42.3years (range 2-98 years). HBV prevalence among those presenting with sign and symptoms of hepatitis was $100(52.1 \%)$ while none of them was positive for HCV. The prevalence of HBV was higher in female 54(56.8\%) and in individual $<30$ years old 31(58.5\%).

Table 1: Demographic characteristics and prevalence study participants ( $N=192)$.

\begin{tabular}{|c|c|c|c|c|c|c|}
\hline Characteristic & Categories & Frequency (\%) & HBV positive cases & HBV negative cases & $\chi^{2}$ & P Values \\
\hline \multirow{2}{*}{ Sex } & Male & $97(50.5)$ & $46(47.4 \%)$ & $51(52.6 \%)$ & \multirow{2}{*}{1.76} & \multirow{2}{*}{0.19} \\
\hline & Female & $95(49.5)$ & $54(56.8 \%)$ & $41(43.2 \%)$ & & \\
\hline \multirow{3}{*}{ Age group } & $<30$ years & $53(27.6)$ & 31 (58.5\%) & $22(41.5 \%)$ & \multirow{3}{*}{2.45} & \multirow{3}{*}{0.29} \\
\hline & 30-49years & $76(39.6)$ & $41(53.9 \%)$ & $35(46.1 \%)$ & & \\
\hline & >50years & $63(32.8)$ & $28(44.4 \%)$ & 35 (55.6) & & \\
\hline
\end{tabular}

\section{Mean (SE) levels of ALT and AST in the study population}

Table 2: Comparison of mean \pm SE ALT and AST levels between HBV infected cases and negative cases.

\begin{tabular}{|c|c|c|c|c|}
\hline Factor & HBV positive cases (n) & HBV negative cases(n) & F & P values \\
\hline ALT(IU/L) & $76.9 \pm 10.6(100)$ & $36.5 \pm 4.7(92)$ & 12.41 & 0.001 \\
\hline AST(IU/L) & $106.7 \pm 14.4(100)$ & $60.7 \pm 9.2(92)$ & 6.81 & 0.010 \\
\hline
\end{tabular}

Table 3: Assessing effect of gender on enzymes value (Mean $\pm \mathrm{SE}$ ) among patients with viral hepatitis.

\begin{tabular}{|c|c|c|c|c|}
\hline \multirow{2}{*}{ Factor } & Indicator & Mean \pm SE & F & P values \\
\hline \multirow{2}{*}{ ALT(IU/L) } & Male & $79.6 \pm 16.8$ & 0.077 \\
\cline { 2 - 4 } & Female & $73.39 \pm 15.3$ & 0.782 \\
\cline { 2 - 5 } AST(IU/L) & Male & $115.6 \pm 21.6$ & 0.672 & 0.415 \\
\cline { 2 - 5 } & Female & $95.9 \pm 19.6$ & \\
\hline
\end{tabular}

SE: Standard Error, AST: Aspartate Aminotransferase; ALT: Alanine Aminotransferase 
Comparison of mean (SE) ALT(IU/L) and AST(IU/L) levels showed a significant $(p<0.05)$ higher values $76.9 \pm 10.6$ and $106.7 \pm 14.4$ respectively in HBV infected subjects than in HBV negative individuals (Table 2). Amongst those with HBV, mean (SE) ALT(IU/L) and AST(IU/L) levels were insignificantly ( $p>0.05)$ higher in male $79.6 \pm 16.8$ and $115.6 \pm 21.6$ than in female (Table 3 ) and in the age range; $>50$ years compare to the other age group $110.6 \pm 31.7$ and $118.4 \pm 24.5$ respectively (Table 4 ). The prevalence of elevated ALT and AST (hepatotoxicity) in the entire study population was
$85(44.3 \%)$ and $68(35.4 \%)$ respectively. There was a significant difference $(\mathrm{p}<0.05)$ seen in the prevalence of elevated AST among HBV negative $25(27.2 \%)$ and HBV positive $60(60.0 \%)$ while that for ALT was 20(21.7\%) for HBV negative and 48(48.0\%) for HBV positive. The prevalence of grade 1 and grade 4 hepatotoxicity were highest and least respectively among those with elevated ALT and AST (Figure 1). These prevalences were highest in HBV positive than HBV negative with a significant differences of $\mathrm{p}<0.05$.

Table 4: Assessing effect of age group on enzymes value (Mean $\pm \mathrm{SE}$ ) among patients with viral hepatitis.

\begin{tabular}{|c|c|c|c|c|}
\hline Factor & Indicator & Mean $\pm S E$ & $\mathbf{F}$ & $P$ values \\
\hline \multirow{3}{*}{ ALT(IU/L) } & $<30$ years & $74.5 \pm 22.3$ & \multirow{3}{*}{0.58} & \multirow{3}{*}{0.56} \\
\hline & 30-49years & $57.6 \pm 7.9$ & & \\
\hline & $>$ 50years & $110.6 \pm 31.7$ & & \\
\hline \multirow{3}{*}{ AST(IU/L) } & $<30$ years & $82.4 \pm 26.4$ & \multirow{3}{*}{1.580} & \multirow{3}{*}{0.21} \\
\hline & 30-49years & $113.4 \pm 28.9$ & & \\
\hline & $>50$ years & $118.4 \pm 24.5$ & & \\
\hline
\end{tabular}

SE: Standard Error; AST: Aspartate Aminotransferase: ALT: Alanine Aminotransferase

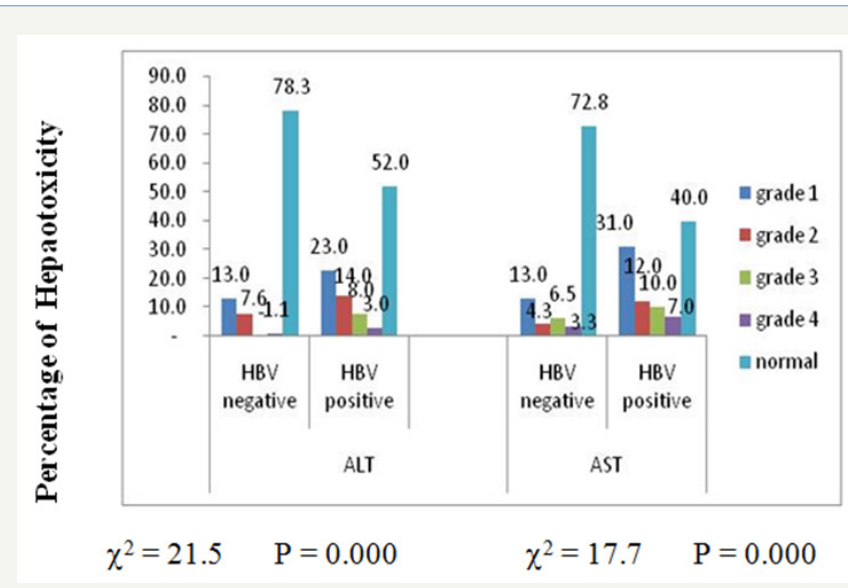

Figure 1: Prevalence (\%) of hepatotoxicity grade among positive and negative HB patients.

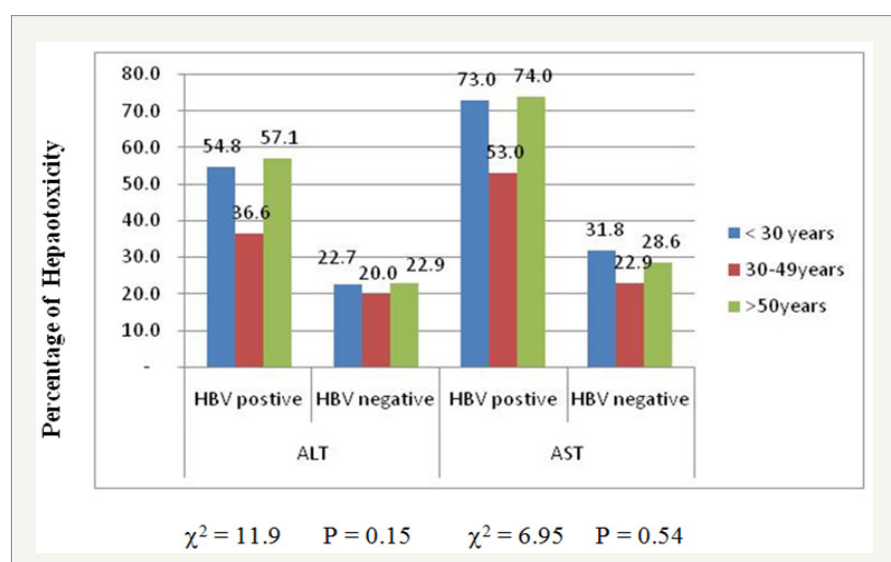

Figure 2: Prevalence (\%) of hepatotoxicity among the different age group.

The highest prevalence of elevated enzymes was seen in the $>50$ years age group $36(67.9 \%)$ while the least was in the $<30$ years age group 39(62.9\%). This differences were not significant $\mathrm{p}>0.58$ with either ALT or AST (Figure 2). Male gender experiences an insignificantly $(\mathrm{p}>0.05)$ higher ALT and AST elevated enzyme compare to their female counterpart $37(38.9 \%)$ and $48(50.5 \%)$ vs $31(32.0 \%)$ and $37(49.5 \%) 54(55.7 \%)$ respectively (Figure 3).

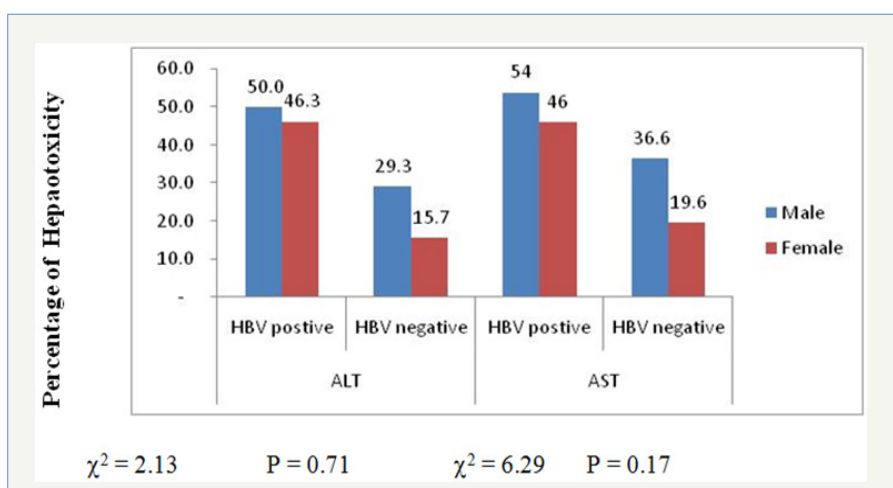

Figure 3: Prevalence (\%) of hepatotoxicity between male and female.

\section{Assessing the type of liver disease}

Table 5: classification liver disease by HB status.

\begin{tabular}{|c|c|c|c|c|}
\hline Categories & $\begin{array}{c}\text { HBV Positive } \\
\text { Cases }\end{array}$ & $\begin{array}{c}\text { HBV Negative } \\
\text { Cases }\end{array}$ & $\chi^{2}$ & P values \\
\hline ALD & $16(16.0 \%)$ & $28(30.4 \%)$ & 5.56 & 0.02 \\
\hline CLD & $84(84.0 \%)$ & $64(69.6 \%)$ & & \\
\hline
\end{tabular}

ALD: Alcoholic Liver Disease; CLD: Chronic Liver Disease

Considering that ratio of AST to ALT 2:1 is suggestive of alcoholic liver disease (ALD) and $<2$ is chronic liver disease (CLD), the prevalence of CLD was higher among HBV patients while ALD was higher in non HBV patients (Table 5). 


\section{Discussion}

The results obtained from this study showed that the HBsAg positivity rate was $100(51.8 \%)$. Average AST and ALT level were higher in HB individual than HB negative individuals similar to report from Achmad et al. [19]. The prevalence of 22(24.0\%) among clients presenting with syndrome of HBV but tested negative for HBV is indicative that other diseases are likely to present similarly to HBV as such all co-morbidity conditions must be fully explored along all detailed list of drugs being taken before drug initiations. This present study confirms the diagnostic usefulness of liver function enzyme as predictor of liver disease in patient.

Similarly, higher ALT and AST values in HBV positive is clear indication that HBV causes injury to the liver, which eventually results in an increase in serum concentrations of AST and ALT $[14,20,21]$. Thus transaminases are sensitive indicators of liver cell injury and are most helpful in recognizing acute hepatocellular diseases such as hepatitis [21].

The mean ALT and AST levels were significantly higher $(\mathrm{p}<0.01)$ in HBV (106.7 vs 76.9) and infected patients than negative controls (60.7 Vs 36.5) respectively. This was similar to results of studies carried in Cameroon by Tufon et al. [16] and can be attributed to cell mediated liver cell damage and not cytopathic effect of the virus [22]. From this study mean values of AST (106.7U/L) and (60.7 U/L) were higher in both HBV positive and negative subjects compared to ALT (76.96 U/L) and (37.61 U/L) respectively. This is indicative that the people are suffering from hepatic cirrhosis as reported by Marghoob et al. [21].

Aminotransferase (AST vs ALT) elevation was more common in men (115.6 vs 79.6) compared to women (95.9 vs73.39). Similar result has been reported by Clark et al. [23]. This can be explained by the high alcohol consumption common in males than in females. Alcohol consumption is prevalent in many countries, and is one of the factors most frequently associated with elevated liver enzyme levels and alcohol-induced liver diseases, such as hepatic steatosis [24]. Our study reveals that there was a high ALT and AST value above the normal unit for those who were negative for HBV. This is expected especially in an era where HIV prevalence is high and especially in Cameroon where the practice of Option B+ during which all persons who test positive for HIV are placed on anti retroviral therapy (ART). ART have shown to cause hepatotoxicity in some HIV clients [25,26].

Elevated values of ALT and AST have also been used to differentiate the pathology of the liver diseases. Chronic liver disease was higher in HBV positive 84(84.0\%) while alcohol liver disease was higher in hepatitis negative 28 (30.4\%) subjects. Cameroon is a country characterized with high alcohol intake [27]. As such the high prevalence of hepatotoxicity in those without HB could be attributed to high alcohol intake or the use of other drugs such as antiretroviral. It has been reported that high alcohol intake can leads to risky behaviour that lead to high transmission HIV [27]. However further investigation on elevated gamma-glutamyl transferase needs to be carried to confirm alcohol liver [28,29].

\section{Conclusion}

Although the cause of hepatotoxicity is multi factorial, it is more common among HB patients. In addition we also found that hepatotoxicity is common in alcoholics. As such management of patients with liver disease should not only rely on the measurements of serum aminotransferases alongside the presence of the disease.

\section{Recommendation}

Appropriate diagnosis prior to treatment should be implemented. All patients with elevated ALT and AST should be screened for both HIV and HB. Health campaign should be given on the adverse effect of alcohol in our community.

\section{Acknowledgment}

We thank the management and staff of the hospitals for their collaboration. Our gratitude also goes to the patients and guardians who did not only give their consent but gave in all the collaborations we needed by paying for the entire tests required.

\section{References}

1. Williams R (2006) Global challenges in liver disease. Hepatology 44(3): 521-526.

2. (2006) World Health Organization: Hepatitis B. Fact sheet no: 204, WHO, Geneva, Switzerland.

3. Ndoe GN, Oumarou JN, Ibrahim KMM, Octavie DMF, Fadimatou HBD, et al. (2016) Viral Hepatitis and Incidence of Hepatotoxicity Cases among Patients Infected with HIV under HAART at the Bertoua Day Hospital. Research in HIV and Retroviral Infections 1(1): 1-8.

4. Sy T, Jamal MM (2006) Epidemiology of hepatitis C virus (HCV) infection. Int J Med Sci 3(2): 41-46.

5. Mueller A, Stoetter L, Kalluvya S, Stich A, Majinge C, et al. (2015) Prevalence of hepatitis B virus infection among health care workers in a tertiary hospital in Tanzania. BMC Infect Dis 15(1): 386.

6. Ul Haq N, Hassali MA, Shafie AA, Saleem F, Farooqui M, et al. (2013) A cross-sectional assessment of knowledge, attitude and practice among hepatitis-b patients in Quetta, Pakistan. BMC Public Health 13(1): 448.

7. Njouom R, Tejiokem MC, Texier G, Fontanet A (2015) Prevalence of hepatitis B, hepatitis C and hepatitis D virus infections in Cameroon: Results from a national population based survey (the ANRS 12289 project). Journal of Viral Hepatitis 22(S2): 1-2.

8. Nerrienet E, Pouillot R, Lachenal G, Njouom R, Mfoupouendoun J, et al. (2005) Hepatitis $C$ virus infection in cameroon: A cohort-effect. Journal of Medical Virology 76(2): 208-214.

9. Laurent C, Bourgeois A, Mpoudi M, Butel C, Mpoudi-Ngolé E, et al. (2007) HIV and hepatitis C virus Coinfection, Cameroon. Emerg Infect Dis 13(3): 514-516.

10. Pépin J, Lavoie M, Pybus OG, Pouillot R, Foupouapouognigni Y, et al. (2010) Risk factors for hepatitis C virus transmission in colonial Cameroon. Clin Infect Dis 51(7): 768-776.

11. Lee LK, Gan VC, Lee VJ, Tan AS, Leo YS, et al. (2012) Clinical relevance and discriminatory value of elevated liver Aminotransferase levels for Dengue severity. PLoS Negl Trop Dis 6(6): e1676.

12. Sulkowski MS (2004) Drug-induced liver injury associated with Antiretroviral therapy that includes HIV-1 protease inhibitors. Clinical Infectious Diseases 38(Suppl 2): S90-S97.

13. Mulu W, Gidey B, Chernet A, Alem G, Abera B, et al. (2013) Hepatotoxicity 
and associated risk factors in Hiv-Infected patients receiving Ethiopia. Ethiop J Health Sci 23(3): 217-226.

14. Giannini EG, Testa R, Savarino V (2005) Liver enzyme alteration. CMAJ 172(3): 367-379. Aminotransferase (AST/GOT) and Alanine Aminotransferase (ALT/GPT) detection techniques. Sensors 6(7): 756-782.

16. Tufon KA, Meriki HD, Anong DN, Mbunkah HA, Nkuo-Akenji T, et al (2016) Genetic diversity, viraemic and aminotransferases levels in chronic infected hepatitis B patients from Cameroon. BMC Res Notes 9: 117.

17. http://www.raosoft.com/samplesize.html

18. Mugusi S, Ngaimisi E, Janabi M, Minzi O, Bakari M, et al. (2012) Liver enzyme abnormalities and associated risk factors in HIV patients on Efavirenz-Based HAART with or without tuberculosis Co-Infection in Tanzania. PLoS One 7(7): e40180.

19. Achmad A, Amalia RF, Mustofa F, Prihadi AL (2014) The incidence of liver fibrosis based on non-invasive markers and hepatotoxic drug used in hepatitis B patients. Asian J Pharm Clin Res 7(5): 287-290.

20. Kim WR, Flamm SL, Di Bisceglie AM, Bodenheimer HC (2008) Serum activity of alanine aminotransferase (ALT) as an indicator of health and disease. Hepatology 47(4): 1363-1370.

21. Marghoob H, Hyder MA, Mohieldein AH (2013) Comparative Levels of ALT, AST, ALP and GGT in Liver associated Diseases. Eur J Exp 3(2): 280284. Antiretroviral therapy at Felege Hiwot referral hospital, Bahirdar,

15. Huang XJ, Choi YK, Im HS, Yarimaga O, Yoon E, et al. (2006) Aspartate

22. Mukherjee RM (2010) Relationship between serum HBsAg level, HBV DNA level, and peripheral immune cells in patients with chronic hepatitis B virus infection. Hepat Med 2: 157-162.

23. Clark J, Brancati FL, Diehl AM (2003) The prevalence and etiology of elevated aminotransferase levels in the United States. Am J Gastroenterol 98(5): 960-967.

24. Park EY, Lim MK, Oh JK, Cho H, Bae MJ, et al. (2013) Independent and supra-additive effects of alcohol consumption, cigarette smoking, and metabolic syndrome on the elevation of serum liver enzyme levels. PLoS One 8(5): e63439.

25. Fokunang CN, Banin AN, Kouanfack C, Ngogang JY (2010) Evaluation of hepatotoxicity and nephrotoxicity in HIV patients on highly active antiretroviral therapy. J AIDS HIV Res 2(3): 48-57.

26. Lucien K, Clement A, Fon N, Weledji P, Ndikvu C, et al. (2010) The effects of antiretroviral treatment on liver function enzymes among HIV-infected out patients attending the central hospital of Yaounde, Cameroon. African Journal of Clinical and Experimental Microbiology 11(3).

27. Kongnyuy EJ, Wiysonge CS (2007) Alcohol use and extramarital sex among men in Cameroon. BMC Int Health Hum Rights 7: 6.

28. Nemat Moussavian S, Becker RC, Piepmeyer JL, Mezey E, Bozian RC, et al. (1985) Serum gamma-glutamyl transpeptidase and chronic alcoholism. Digestive Diseases and Sciences 30(3): 211-214.

29. http://www.who.int/mediacentre/factsheets/fs204/en. assessed on the $13 / 12 / 16$

\section{Your subsequent submission with Crimson Publishers} will attain the below benefits

Creative Commons Attribution 4.0

International License

For possible submission use the below is the URL 